\footnotetext{
1. FCPS (Cardiology)

Fellow Interventional Cardiology AFIC/NIHD.

2. FCPS (Med)

Registrar

MTI ATH.

3. FCPS

Fellow Interventional Cardiology AFIC/NIHD.

4. MBBS

Student Final Year MBBS

AMC.

5. MBBS

Student Final Year MBBS

AMC.

6. MBBS

Student Final Year MBBS

AMC.

7. FCPS (Card)

Fellow Interventional Cardiology NICVD.
}

Correspondence Address:

Dr. Ariz Samin

Fellow Interventional Cardiology AFIC/NIHD.

dr_ariz@yahoo.com

Article received on:

12/09/2019

Accepted for publication:

02/09/2020

\section{In-hospital mortality in patients with non-st segment elevation myocardial infarction in relation to high risk global registry of acute cardiac events score.}

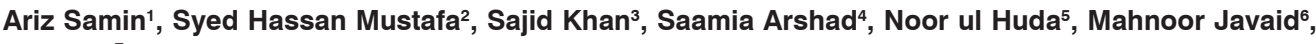
Nasir Ali $^{7}$

ABSTRACT... Objectives: the purpose of this study was to validate GRACE scoring as a tool for predicting both short and long term outcomes in patients with NSTEMI. Study Design: Descriptive Case Series. Setting: Department of Cardiology Ayub Teaching Hospital, Abbottabad. Period: 4th August 2016 to 4th April 2017. Material \& Methods: 199 patients diagnosed with NSTEMI were included in the study after obtaining an apprised consent. Risk stratification of each patient was done according to GRACE score. Patients were followed up during their hospital stay and their outcome was recorded on a pre-designed pro forma. The outcome was described as either death or discharge. Results: Mean \pm SD GRACE score was 156.12 \pm 20.65 . The overall mortality in the study population was $11.6 \%(n=23)$. When the outcome variable was stratified according to age, gender, diabetes mellitus, obesity and hypertension, results were found in case of hypertension $(p<0.05)$, and statistically no significant in the case of other variables. Conclusion: A high risk GRACE score is associated with increased in-hospital mortality in patients with NSTEMI.

Key words: $\quad$ Acute Coronary Syndrome, GRACE, NSTEMI, TIMI.

Article Citation: Samin A, Mustafa SH, Khan S, Arshad S, Noor ul Huda, Ali N, Javaid M. In-hospital mortality in patients with NON-ST segment elevation myocardial infarction in relation to high risk global registry of acute cardiac events score. Professional Med J 2020; 27(11):2433-2437.

https://doi.org/10.29309/TPMJ/2020.27.11.4146

\section{INTRODUCTION}

The most important medical and public health issue is Coronary artery disease (CAD) which having death rate of about 7.2 million throughout the world per year, which approximates about $12 \%$ globally. This disease is also considered as the most important and single most cause of mortality in third world countries. ${ }^{1,2}$ Most of the patients presented at emergency unit having already coronary artery disease is suffered from Acute Coronary syndrome (ACS). The ACS symptoms resembled the clinical symptoms of acute myocardial ischemia. The terminology of Syndrome has been given to this entity because of its different mode of presentations. These presentations are named like unstable angina, non-ST elevation myocardial infarction (NSTEMI) and ST-elevation myocardial infarction (STEMI). These are named on the basis of symptoms, electrocardiographic findings and cardiac markers. ${ }^{3}$
If there is only changes in the cardiac markers on laboratory reports but the electrocardiography having no changes in the ST segement in tow or more continuous leads is named as NSTEMI ${ }^{4}$ Among all entities of Coronary Artery Syndrome, the entity which having highest mortality is NSTEMI. ${ }^{5}$ It having the highest mortality because it occur in patients with severe artery stenosis, transient occlusion, atheroma formation and having micro emboli or thrombus. ${ }^{6}$ The emergency diagnosis of such patients should be done as soon as possible to decrease the mortality rate as well as to start early different management therapies and restore early coronary blood flow.

The patients who having coronary artery disease and are risk of such syndrome can be diagnosed through different scoring systems. Among them, the most commonly used are "Thrombolysis in Myocardial Infarction risk scores (TIMI) and Global Registry of Acute Cardiac Events (GRACE). ${ }^{7}$ 
GRACE risk score (GRS) was developed from the registry, which has been proven beneficial in predicting both short and long term death and major cardiac event. ${ }^{3}$ The components of the GRACE Risk Score (range 1-263) include age, systolic blood pressure, heart rate, Killip class, history of cardiac arrest, renal functions, STsegment deviation, and cardiac biomarkers.

GRACE risk score (GRS) has been used to predict in-hospital mortality of NSTEMI patients. For instance, according to a study, in GRACE registry the in-hospital mortality was found to be $4 \%$ for NSTEMI. ${ }^{8}$ Another study showed that hospital death rates for NSTEMI corresponding with high risk GRACE risk score was $9.1 \%{ }^{9}$

It is very important to accurately predict the long term outcome of patients that presents to the hospital with NSTEMI. GRACE scoring provides help in not only predicting long term outcomes, but also helps in early risk stratification. The occurrence of such lethal condition can be decreased through this GRACE scoring system. ${ }^{10}$

Therefore the purpose of this study was to validate GRACE scoring as a tool for predicting both short and long term outcomes in patients with NSTEMI. It also guides to select early invasive or early conservative strategy. GRACE study has not yet been tested on our population.

\section{MATERIAL \& METHODS}

This descriptive, case series was done from $4^{\text {th }}$ August 2016 to $4^{\text {th }}$ April 2017 in the Cardiology department of Ayub Teaching Hospital, Abbottabad. A sample of 199 patients were included in the study through non-probability consecutive sampling, calculated by using the WHO software using $9.1 \%$ as frequency of Inhospital mortality in patients with NSTEMI in relation to high risk GRACE. ${ }^{8}$ A confidence level of $95 \%$ and absolute precision of $4 \%$ was used. Only patients aged between 18 and 80 years and with GRACE score of more than 140 were included in the study. Similarly, patients having ST-segment elevation or new onset left bundle branch block on admission were excluded from the study to control confounding.
After getting permission from the hospital ethics committee, data was collected from all newly diagnosed NSTEMI patients. An informed written consent was taken from the patient. A detailed history was taken and complete physical examination was performed including body measurements like height and weight. Obesity was defined as having a Body Mass Index (BMI) of more than 30. Risk stratification of each patient was carried out by asking questions related to the presence or absence of risk indicators which are also part of GRACE risk score. In this way each patient was scored according to number of GRACE risk factors present. Data of all patients with a risk score of 140 or more was collected using a pre-designed pro forma. Data was entered into and analyzed using software SPSS version 20.0. Quantitative variables like age, weight, height, BMI, and GRACE score were described as mean $\pm \mathrm{SD}$. Categorical variables like gender, presence of diabetes, hypertension and death due to NSTEMI were described as frequencies and percentages. Outcome variables were stratified by age, gender, diabetes and obesity and hypertension. To know significance difference between age, gender, diabetes, hypertension and obesity with respect to outcome variables, chi square test at $5 \%$ significance level was used.

\section{RESULTS}

The sample comprised of 199 patients. Of these 127 (63.8\%) were males and 72 (36.2\%) were females. Age ranged from 31 to 70 with mean \pm SD of $57.61 \pm 9.33$ years. Likewise, the weights and heights of the patients ranged from 48 to 100 kilograms and 1.5 to 1.8 meters, with mean \pm SD as $70.65 \pm 10.94$ kilograms and $1.65 \pm 0.75$ meters respectively. The mean \pm SD body mass index of study participants was $26.06 \pm 3.98$ ranging from 17.28 to 39.06 .

Only high-risk GRACE score (>140) patients were recruited. In the patient population 30 (11.6\%) persons died during the in-hospital stay.

The mean \pm SD GRACE Score of study participants was 156.12 \pm 20.65 . The GRACE Risk Score showed good discrimination, with area under the ROC curve of 0.91 (95\% Cl 0.85-0.97, $P=0.000)$. The ROC curve was characteristic in 
this study $(p=0.000)$.

The observed mortality in the study cohort was $11.6 \%$ (23). When the outcome variable, i.e. mortality was stratified by age, gender, presence of diabetes and obesity, the differences were statistically not significant $(p>0.05)$, whereas hypertension among study participants was found significantly associated with mortality $(p<$ 0.05). Some results are shown in the tables.

\begin{tabular}{|c|c|c|}
\hline \multicolumn{2}{|c|}{ Age of the Patients } & \multirow{2}{*}{ P-Value } \\
\hline$\leq 55$ Years & $>55$ Years & \\
\hline 10 & 13 & 0.94 \\
\hline \multicolumn{2}{|c|}{ Gender of the Patient } & \\
\hline Male & Female & \\
\hline 17 & 6 & 0.28 \\
\hline \multicolumn{2}{|l|}{ Diabetes } & \\
\hline Present & Absent & \\
\hline 10 & 13 & 0.11 \\
\hline \multicolumn{2}{|l|}{ Obesity } & \\
\hline Present & Absent & \\
\hline 4 & 19 & 0.74 \\
\hline \multicolumn{2}{|l|}{ Hypertension } & \\
\hline Present & Absent & \\
\hline 21 & 2 & 0.009 \\
\hline
\end{tabular}

\section{DISCUSSION}

Acute coronary syndrome has been relegated into STEMI and NSTEMI, and a number of risk scores have been designed to help predict their outcome. Among them, the most commonly used risk score is the GRACE risk score (GRS). ${ }^{11}$

Our study showed a vigorous relationship of high GRACE score with in hospital mortality. The mean \pm SD GRACE Score of study participants was 156.12 \pm 20.65 . The GRACE Risk Score showed good discrimination, with area under the ROC curve of 0.91 (95\% Cl 0.85-0.97, $\mathrm{P}=0.000)$. During the in-hospital stay, 30 (11.3\%) patients died. This study fixated on in-hospital mortality ergo, deaths occurring later on were not recorded. Similar results have been reported recently by Shaikh and colleagues from Karachi, Pakistan. ${ }^{12}$
The researchers sought to validate the Grace Risk Score as a tool to predict the short term and long term outcome of patients with NSTEMI and unstable angina. In one of their observational study, they recruited 530 patients and calculated GRACE risk score for every patient. Then, every patient was grouped either into high, intermediate or low risk groups according to their GRS. The overall mean grace risk score in our population was $131.87+/-41.56$. The GRACE Risk Score showed good discrimination, with Area under the ROC curve of $0.803(95 \% \mathrm{Cl} 0.705-0.902, \mathrm{P}$ $<0.001)$. During the in-hospital stay, total of 19 (3.6\%) patients died, and out of those 15 (8.4\%) patients belonged to high risk group. ${ }^{12}$ In contrast to this, our study only recruited patients with a high-risk GRACE score (>140). The total mortality in the patient population was $11.6 \% \quad(n=30)$. However, the ROC curve was characteristic in this study $(p=0.000)$.

Similarly, Koonsiripaiboon and Tungsubutra withal sought to validate GRACE score in prognostication of in-hospital mortality in patients with STEMI. ${ }^{13}$ They included 209 patients of STEMI in their study and calculated GRACE scoring of every patient. They then classified their patients into three groups based on GRACE score; high, intermediate and low risk groups. Assessment of in-hospital mortality was done and comparison with GRACE scoring was made. The mean GRS was $161 \pm 46.2$ and the overall in-hospital mortality was $12.4 \%$. However, mortality in high risk group was reported to be $23.3 \%(95 \% \mathrm{Cl} 16.2-32.3) .{ }^{13}$

The mortality rates reported by the researcher are much higher than observed in our study. This may be because patients with STEMI were included in this study which has a scarcely higher rate of complications. Supplementally, albeit the sample size was virtually kindred to this study $(n=209)$, they divided the patients into three categories of low-, medium- and high GRACE risk score. On the other hand, our study only included patients with high-risk GRACE score and those with NSTEMI, consequently the results of these studies cannot be compared with each other. Withal in Koonsiripaiboon et al, diabetes was not associated with high mortality which was in 
accordance with our study..$^{13}$

Caution should be exercised afore generalization of the results to the whole population as this is a diminutive hospital predicated study with a diminutive sample size. In integration, only patients with high risk GRACE score were recruited, consequently, sodality of mortality with the high risk GRACE score cannot authentically be ascertained because no data was accumulated for low- and medium-risk GRACE scores and we observed only in-hospital mortality, and no longterm follow-up of the patients was studied.

\section{CONCLUSION}

GRACE score should be calculated for every patient admitted with NSTEMI. Albeit more research is needed to determine the role played by presence of chronic disease states such as diabetes mellitus, hypertension and dyslipidemias, an elevated high-risk GRACE score is associated with incremented in-hospital mortality in patients admitted with NSTEMI.

Copyright@ 02 Sep, 2020.

\section{REFERENCES}

1. Masic I, Rahimic M, Dilic M, Kadribasic R, Toromanovic $\mathrm{S}$. Socio-medical characteristics of coronary disease in Bosnia and Herzegovina and the world. Mater Sociomed 2011; 23(3):171-83.

2. John FB, Rachel D, Rosanna T. Epidemiology of Coronary Artery Disease. Coronary Artery Disease Current Concepts in Epidemiology, Pathophysiology, Diagnostics and Treatment. ISBN: 978-953-51-0262-5, InTech 2012, Available from: http://www.intechopen. com/ books/coronary-artery-disease-current-conceptsinepidemiology-pathophysiologydiagnostics-andtreatment/ epidemiology-of-coronary-artery-disease.

3. Zhou BD, Zu LY, Mi L, Wang GS, Guo LJ, Gao W. An analysis of patients receiving emergency CAG without $\mathrm{PCl}$ and the value of GRACE score in predicting $\mathrm{PCl}$ possibilities in NSTE-ACS patients. $\mathrm{J}$ Geriatr Cardiol. 2015; 12(3):246-50.

4. Cannon C, Braunwald E. Unstable angina and nonST elevation myocardial infarction. Braunwald's Heart Disease: A Textbook of Cardiovascular Medicine [Internet]. Elsevier - Health Sciences Division; 2012. p. 1178-209. (Braunwald's Heart Disease: A Textbook of Cardiovascular Medicine). Available from: https:// books.google.com.pk/books?id=blq42K8AY5AC.
5. Meier P, Lansky AJ, Baumbach A. Almanac 2013: Acute coronary syndromes. Heart. 2013; 99(20):1488-93.

6. Daga LC, Kaul U, Mansoor A. Approach to STEMI and NSTEMI. J Assoc Physicians India. 2011; 59 Suppl:19-25.

7. Ascenzo DF, Zoccai BG, Moretti C, Bollati M, Omedè P, Sciuto F, et al. TIMI, GRACE and alternative risk scores in Acute Coronary Syndromes: A meta-analysis of 40 derivation studies on 216,552 patients and of 42 validation studies on 31,625 patients. Contemp Clin Trials. 2012; 33(3):507-14.

8. Prabhudesai AR, Srilakshmi MA, Santosh MJ, Shetty $\mathrm{GG}$, Varghese $\mathrm{K}$, Patil $\mathrm{CB}$, et al. Validation of the GRACE score for prognosis in Indian patients with acute coronary syndromes. Indian Heart J. 2012; 64(3):263-9.

9. Abu-Assi E, Ferreira-González I, Ribera A, Marsal JR, Cascant P, Heras M, et al. Do GRACE (Global Registry of Acute Coronary events) risk scores still maintain their performance for predicting mortality in the era of contemporary management of acute coronary syndromes? Am Heart J. 2010; 160(5):826-834

10. Harvey J. Unstable angina and non-ST elevation myocardial infarction. In: Griffin BP, Callahan T, Menon V. Manual of Cardiovascular Medicine [Internet]. Wolters Kluwer Health; 2012. p. 36-59. Available from: https:// books.google.com.pk/books?id=7t1lfP37kkYC.

11. Pedro de AG, Jorge F, Carlos A, Ricardo SG. TIMI, PURSUIT, and GRACE risk scores: Sustained prognostic value and interaction with revascularization in NSTE-ACS. Eur Heart J. 2005; 26:865-872.

12. Shaikh MK, Hanif B, Shaikh K, Khan W, Parkash J. Validation of grace risk score in predicting in1hospital mortality in patients with non ST-elevation myocardial infarction and unstable angina. J Pak Med Assoc. 2014 Jul; 64(7):807-11.

13. Koonsiripaiboon E, Tungsubutra W. Validation of the GRACE risk score to predict in-hospital mortality in patients with ST segment elevation myocardial infarction in Thailand. J Med Assoc Thai. 2013; 96(2):139-45. 


\section{AUTHORSHIP AND CONTRIBUTION DECLARATION}

\begin{tabular}{|c|c|c|c|}
\hline Sr. \# & Author(s) Full Name & Contribution to the paper & Author(s) Signature \\
\hline 1 & Ariz Samin & $\begin{array}{l}\text { Data collection, Paper writing } \\
\text { and supervision. }\end{array}$ & \\
\hline 2 & Syed Hassan Mustafa & Paper writing. & \\
\hline 3 & Sajid Khan & Paper writing. & \\
\hline 4 & Saamia Arshad & Data collection & \\
\hline 5 & Noor ul Huda & Data analysis & \\
\hline 6 & Mahnoor Javaid & Data analysis & \\
\hline 7 & Nasir Ali & Data collection & \\
\hline
\end{tabular}

